\title{
Trivial Excitation Energy Transfer to Carotenoids Is an Unlikely Mechanism for Non-photochemical Quenching in LHClI
}

\author{
Callum Gray ${ }^{1}$, Tiejun Wei ${ }^{1}$, Tomáš Polívka ${ }^{2}$, Vangelis Daskalakis ${ }^{3}$ and \\ Christopher D. P. Duffy ${ }^{1 *}$
}

\begin{abstract}
${ }^{1}$ Digital Environment Research Institute (DERI), Queen Mary University of London, London, United Kingdom, ${ }^{2}$ Department of Physics, Faculty of Science, University of South Bohemia, Ceske Budejovice, Czechia, ${ }^{3}$ Department of Chemical Engineering, Cyprus University of Technology, Limassol, Cyprus
\end{abstract}

\section{OPEN ACCESS}

Edited by:

Eunchul Kim,

Graduate University for Advanced

Studies, Japan

Reviewed by:

Thomas Renger,

Johannes Kepler University of Linz,

Austria

Yutaka Shibata,

Tohoku University, Japan

Ivo H. M. Van Stokkum,

VU Amsterdam, Netherlands

*Correspondence:

Christopher D. P. Duffy

c.duffy@qmul.ac.uk

Specialty section:

This article was submitted to

Plant Physiology

a section of the journal

Frontiers in Plant Science

Received: 18 October 2021 Accepted: 20 December 2021

Published: 13 January 2022

Citation:

Gray C, Wei T, Polívka T, Daskalakis V and Duffy CDP (2022) Trivial Excitation

Energy Transfer to Carotenoids Is an Unlikely Mechanism for

Non-photochemical Quenching in LHCII. Front. Plant Sci. 12:797373.

doi: $10.3389 /$ fpls. 2021.797373
Higher plants defend themselves from bursts of intense light via the mechanism of Non-Photochemical Quenching (NPQ). It involves the Photosystem II (PSII) antenna protein $(\mathrm{LHCll})$ adopting a conformation that favors excitation quenching. In recent years several structural models have suggested that quenching proceeds via energy transfer to the optically forbidden and short-lived $S_{1}$ states of a carotenoid. It was proposed that this pathway was controlled by subtle changes in the relative orientation of a small number of pigments. However, quantum chemical calculations of $S_{1}$ properties are not trivial and therefore its energy, oscillator strength and lifetime are treated as rather loose parameters. Moreover, the models were based either on a single LHCII crystal structure or Molecular Dynamics (MD) trajectories about a single minimum. Here we try and address these limitations by parameterizing the vibronic structure and relaxation dynamics of lutein in terms of observable quantities, namely its linear absorption (LA), transient absorption (TA) and two-photon excitation (TPE) spectra. We also analyze a number of minima taken from an exhaustive meta-dynamical search of the LHCll free energy surface. We show that trivial, Coulomb-mediated energy transfer to $S_{1}$ is an unlikely quenching mechanism, with pigment movements insufficiently pronounced to switch the system between quenched and unquenched states. Modulation of $S_{1}$ energy level as a quenching switch is similarly unlikely. Moreover, the quenching predicted by previous models is possibly an artifact of quantum chemical over-estimation of $S_{1}$ oscillator strength and the real mechanism likely involves short-range interaction and/or non-trivial inter-molecular states.

Keywords: carotenoid, non-photochemical quenching (NPQ), LHCIl, energy-dissipation, photosystem (PSII), transient absorption

\section{INTRODUCTION}

Non-photochemical quenching (NPQ) in higher plants is a regulatory response to a sudden increase in light intensity (Horton et al., 2000; Niyogi, 2000; Müller et al., 2001; Ruban et al., 2012). It is a (mostly Malnoë et al., 2018) reversible down-regulation of the quantum efficiency of the Photosystem II (PSII) lightharvesting antenna (LHCII) with the purpose of defending the saturated reaction centers from over-excitation and photoinhibition (Powles, 1984; Aro et al., 1993). 
Essentially, it is due to the creation of exciton-quenching species within LHCII which trap and dissipate chlorophyll excitation before it can accumulate in PSII and damage the reaction centers. While the fine molecular details of the mechanism are still unclear, a general consensus has emerged over the basic scheme. The primary trigger of NPQ is an acidification of the thylakoid lumen $(\Delta \mathrm{pH})$ due to a high rate of electron transport (Strand and Kramer, 2014), in large part arising from cyclic electron flow about PSI (Sato et al., 2014). The $\triangle \mathrm{pH}$ activates three components of NPQ: the PSII antenna sub-unit PsbS (Li et al., 2004), the enzyme violaxanthin deepoxidase (VDE) (Jahns et al., 2009), and the LHCII antenna proteins themselves (Walters et al., 1994; Liu et al., 2008; Belgio et al., 2013). VDE converts the violaxanthin pool to zeaxanthin which may lead to violaxanthin-zeaxanthin exchange in the loose, peripheral xanthophyll-binding site of LHCII (Xu et al., 2015). It has been shown that the presence of zeaxanthin affects the kinetics and amplitude of NPQ but is not a strict requirement for it (Ruban and Horton, 1999; Nicol and Croce, 2021). Quenching can similarly be achieved in the absence of PsbS if $\Delta \mathrm{pH}$ is driven to non-physiological levels (Johnson and Ruban, 2011). Either way (for further information, the reader is directed to a comprehensive review of this complex and ongoing topic Ruban, 2016) the combined effect is to induce an in-membrane aggregation or clustering of LHCII (Horton et al., 1991) and some subtle internal conformational changes (Ilioaia et al., 2011). These somehow modulate the pigment-pigment and pigment-protein couplings to create a quenching species, although the nature of the quencher and molecular dynamics of the conformational "switch" are still unclear.

Recently, it has become broadly (though by no means universally) accepted that the quencher is or involves one of the LHCII carotenoid (Cart) pigments (Ma et al., 2003). These are attractive candidates as they are, in a sense, intrinsically quenched, possessing a very short $(\approx 10 \mathrm{ps})$ excitation lifetime relative to chlorophyll ( $\mathrm{Chl}-\approx 4-6 \mathrm{~ns})$. Various mechanisms have been suggested, such as excitation energy transfer (EET) to the Cart which quenches simply by virtue of its short lifetime (Ruban et al., 2007); mixing of the Chl and Cart lifetimes brought on by excitonic resonance (Bode et al., 2009; Holleboom and Walla, 2014); and formation of fast-relaxing ChlCart CT states (Holt et al., 2005; Ahn et al., 2008). The lutein (Lut) bound to the L1 binding site (Wei et al., 2016) of the LHCII trimer is often cited (Ruban et al., 2007) as being the particular carotenoid involved in quenching, but zeaxanthin at an equivalent site in one of the minor PSII antennae has also been proposed (Ahn et al., 2008). We also note that Holzwarth and co-workers present a Cart-independent quencher model that involves Chl-Chl CT states (Müller et al., 2010; Ostroumov et al., 2020). The differences between these models often come down to specific interpretations of highly-congested timeresolved spectral measurements on these complexes. Moreover, any involvement of the Carts is obscured by the fact that their lowest singlet excitation, $S_{1}$, is optically forbidden and decays very quickly (Polívka and Sundström, 2004).

The X-ray structure of LHCII (Liu et al., 2004) can provide some insight into the quencher, particularly since it was found to correspond to a highly dissipative configuration, meaning it could serve as a model structure for the quenched state (Pascal et al., 2005). Several detailed models of this structure very accurately predicted the steady state and time-resolved spectra of LHCII (Novoderezhkin et al., 2004, 2011; Müh et al., 2010) but they did not capture the dissipative character (in fairness that was never their goal). One possible reason for this was their neglect of the Carts, due to the fact that they contribute nothing to the spectrum in the red region and that there are no truly reliable methods for calculating the excitation energy and one-electron transition density of the $S_{1}$ state. The latter is due to the strong electron correlations giving it a complex multi-electron character (Tavan and Schulten, 1987; Andreussi et al., 1993). Beginning in 2013 Duffy and co-workers used a semi-empirical quantum chemistry method to estimate the $S_{1}$ transition density and its potential effect on the excitation lifetime of the LHCII crystal structure (Duffy et al., 2013; Chmeliov et al., 2015; Fox et al., 2017, 2018). These models suggested that quenching was due to EET from the Chl $Q_{y}$ band to the $S_{1}$ state of the centrally bound Luts (L1 and L2), followed by fast decay of $S_{1}$. This EET was mediated by weak resonance couplings between $Q_{y}$ and $S_{1}$ (due to the latter's lack of oscillator strength) and was therefore assumed to be incoherent (Förster transfer) and slow (20-50 ps) relative to excitation equilibration across the Chls $(\approx 1-2 \mathrm{ps})$. This is essentially the mechanism proposed by Ruban et al. based on global target analysis of transient absorption (TA) measurements on LHCII aggregate, although they propose L1 as the sole quencher (Ruban et al., 2007). Of course these models are all based on a single, timeaveraged structural configuration, and a highly artificial one at that. It therefore tells us nothing about how such quenching is switched on and off and can only very tentatively be applied to the actual in vivo quenching mechanism. More recently, the model was extended to a molecular dynamics simulation of the LHCII trimer within a lipid bilayer (Balevičius et al., 2017). Although a stable, unquenched conformation was not identified, it predicted that the $Q_{y}-S_{1}$ coupling was highly sensitive to very small changes in inter-pigment orientations, suggesting that the lifetime could be modulated by very subtle conformational changes. Unfortunately, this appears to have been incorrect for two reasons. Firstly, the coupling sensitivity appears to have been an artifact of the semi-empirical Hamiltonian used to calculate $S_{1}$. Khokhlov and Belov showed that this sensitivity disappears when more rigorous methods are used (Khokhlov and Belov, 2019). Moreover, by simulating the near-identical CP29, Lapillo et al. showed that even with the semi-empirical method, large lifetime fluctuations are significantly dampened if one accounts for the excitonic structure of the Chl manifold in the complex (the previous model assumed a Chl-Lut dimer embedded in some coarse-grained, iso-energetic Chl pool) (Lapillo et al., 2020). In addition to these errors, the model has a series of weaknesses that here we attempt to address:

- The $S_{1}$ excitation energy is neither easy to measure directly or calculate. Transient absorption in near-IR gives a phononless excitation energy of $14,050 \pm 300 \mathrm{~cm}^{-1}$ for Lut in recombinant LHCII (Polívka et al., 2002), while two-photon excitation 
(TPE) in native LHCII gave $<15,300 \mathrm{~cm}^{-1}$ (Walla et al., 2000). The latter value is likely the first vibronic peak which is $\approx$ $1100 \mathrm{~cm}^{-1}$ higher than the phononless peak, meaning the two values agree reasonably well. Nevertheless, it is often treated as a free parameter and large changes to its value have been proposed as a part of the NPQ switch (Holleboom and Walla, 2014; Lapillo et al., 2020).

- The vibronic structure and relaxation dynamics of $S_{1}$ were not properly considered. It was treated as a single optical transition with a line-broadening function chosen to provide a convincing visual fit to the TPE spectrum which implied very large reorganization energies. It was assumed that reorganization on $S_{1}$ was instantaneous and that internal conversion (IC) to the ground state $\left(S_{0}\right)$ occurred with a single rate constant of $\approx 10-20$ ps (Polívka et al., 2002). The end result is a picture of $S_{1}$ as an deep, irreversible trap. In reality, $S_{1}$ is composed of several vibronic transitions that could couple differently to $Q_{y}$, relax on finite timescales and undergo IC at different rates.

- Limited sampling of the LHCII potential energy surface (PES) means we might not be probing biologically relevant conformations. Unsteered MD simulations start from a quenched minimum close to the crystal structure. Single molecule spectroscopy has shown that LHCII trimers will spontaneously switch between quenched and unquenched states but the typical dwell time in each is of the order 1-10 s (Krüger et al., 2010), meaning prohibitively long unsteered simulations may be needed to capture this switching.

Here we attempt to correct these errors in several ways. We obtain a detailed picture of the $S_{1}$ energy gap, vibronic structure and relaxation kinetics by fitting a detailed model to the TA kinetics of Lut in pyridine. These parameters (along with a secular Redfield model of the Chl manifold Malý et al., 2016) are then used to model energy relaxation in LHCII. The LHCII model structures that we use come from an exhaustive steered search of the LHCII PES which was previously published (Daskalakis et al., 2020). The motivation is to determine whether NPQ can realistically be switched on and off simply by altering the relative distance/orientation of Lut and its neighboring chlorophylls.

\section{RESULTS}

\subsection{Steady-State Spectra of the Chlorophyll Excitonic Manifold}

The Chl-Chl relaxation dynamics are modeled according to the method in Malý et al. (2016) and briefly recapped in the Methods. For a given LHCII monomer trajectory we take a set of uncorrelated snapshots and for each calculate the population relaxation. The snapshots sample disorder in the inter-pigment excitonic couplings and the different minima in the original steered MD may reveal differences in the average couplings. We do not calculate the Chl excitation (site) energies in situ but simply take the average values reported in Müh et al. (2010). The reason for this is partly to spare computational expense and partly because these fluctuations have almost no effect on quenching (Balevicius and Duffy, 2020). To check the validity of the model we calculate the linear absorption (LA) and fluorescence (FL) profiles, examples of which are shown in Figures 1A,B, adding Gaussian disorder to the site energies to reproduce the broadening.

\subsection{Relaxation Kinetics of Lutein in Pyridine}

For Lut we adopt the Vibrational Energy Relaxation Approach (VERA) (Balevičius et al., 2018; Balevicius et al., 2019) to reproduce several independent spectral measurements. The details are discussed in Methods (and section C of the Supporting Information) but essentially the four singlet electronic states $\left(\left|S_{0}\right\rangle,\left|S_{1}\right\rangle,\left|S_{2}\right\rangle,\left|S_{n}\right\rangle\right)$ are replaced by sets of vibronic states, $\left(\left|i_{a_{1} a_{2}}\right\rangle=|i\rangle\left|a_{1}\right\rangle^{i}\left|a_{2}\right\rangle^{i}\right)$, where $i$ is the electronic index and $a_{1}$ and $a_{2}$ are the vibrational quantum numbers associated with the high-frequency, optically-coupled $C-C$ and $C=C$ modes, respectively (Balevičius et al., 2018). For example, the state $\left|0_{00}\right\rangle$ corresponds to the absolute ground state and $\left|1_{10}\right\rangle$ to the first excited vibrational state of the $\mathrm{C}$-C stretching mode on the first excited electronic state $\left|S_{1}\right\rangle$. The LA is given by the sum of all vibronic transitions belonging to $\left|S_{0}\right\rangle \rightarrow\left|S_{2}\right\rangle$ (weighted by the Franck-Condon overlaps and the initial populations on $\left|0_{a_{1}, a_{2}}\right\rangle$ ) and is shown in Figure 1C alongside the experimental profile for Lut in pyridine. The fit is very good up to the blue edge of the first vibronic peak after which there is a deviation due to contributions from different geometrical conformers that are not accounted for in our model (Lukeš et al., 2011). The rise above $27,500 \mathrm{~cm}^{-1}$ is a solvent artifact.

The static properties of $S_{1}$ and all dynamical properties were obtained by fitting the VERA model to the TA of Lut in pyridine. Figures $\mathbf{2 A}, \mathbf{B}$ show the calculated and experimental difference spectra at intermediate $(1-20 \mathrm{ps})$ and long $(10-42 \mathrm{ps})$ delay times, respectively. The sub-picosecond kinetics are not shown as they are less relevant to the final quenching model. The $S_{1}$ Excited State Absorption (ESA, positive feature around $18,000 \mathrm{~cm}^{-1}$ ) is well fit but there is some discrepancy for the Ground State Bleach (GSB, negative feature) at earlier times. While the fit can be improved by adjusting the $S_{2}$ parameters, this disrupts the original LA fit. This could be linked to GSBdistorting local heating effects which have previously been reported (Balevicius et al., 2019) or simply an artifact. Either way it is the $S_{1}$ parameters and kinetics that we are primarily interested in. All fitting parameters are reported in section A of the Supporting Information but there are a few key quantities:

- The phononless $S_{1}$ energy, $\varepsilon_{S_{1}}=14,050 \mathrm{~cm}^{-1}$ : We assumed the previously reported value during the fit to reduce the number of free parameters. Varying $\varepsilon_{S_{1}}$ naturally ruins the fit but it can be recovered by adjusting other parameters (mainly $\varepsilon_{S_{n}}$ and the dimensionless displacements between $S_{1}$ and $S_{0}$. As an independent check we calculated the $S_{0} \rightarrow S_{1}$ lineshape and compared it to the TPE of Lut in octanol (Walla et al., 2002). This is shown in Figure 1D and apart from a $150 \mathrm{~cm}^{-1}$ blue shift to account for the different solvent there is a reasonable visual agreement. However, we must note that the fit (nor the data, really) does not match the mirror image of the $S_{1}$ FL line-shapes observed for Carts such as neurosporene and 
A

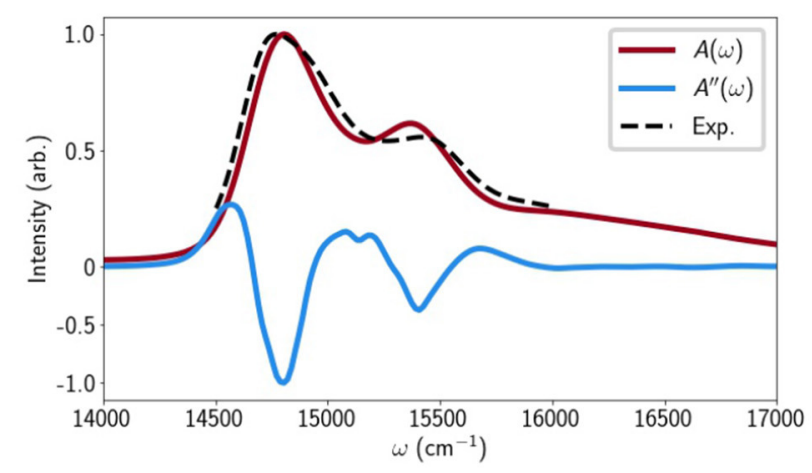

C

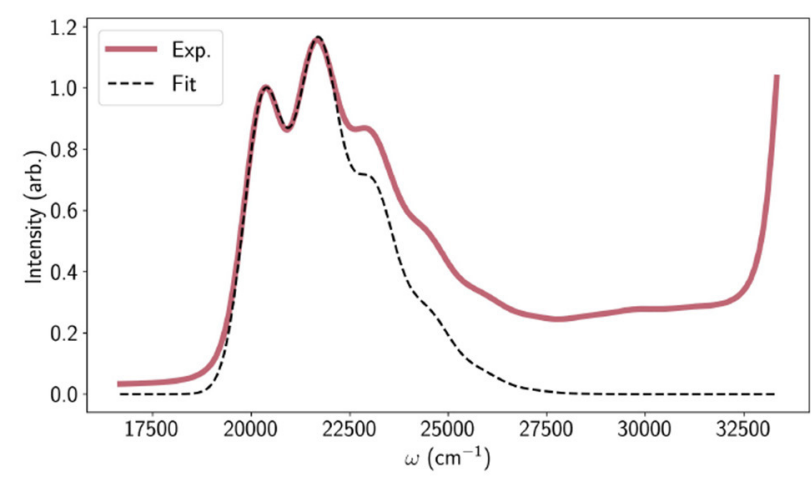

B

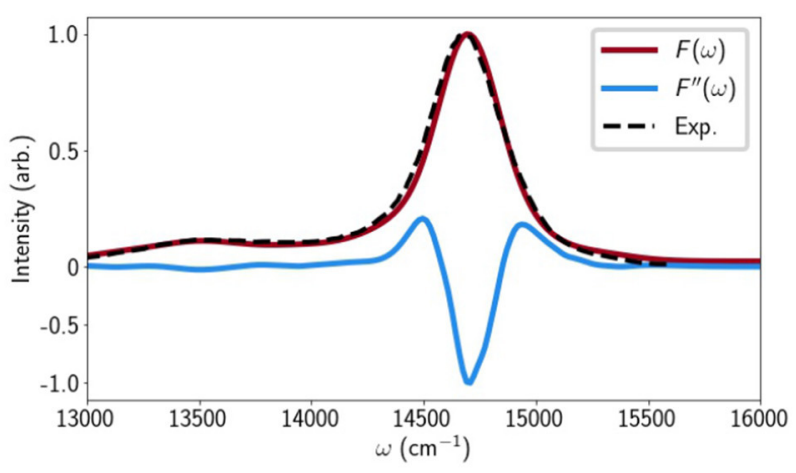

D

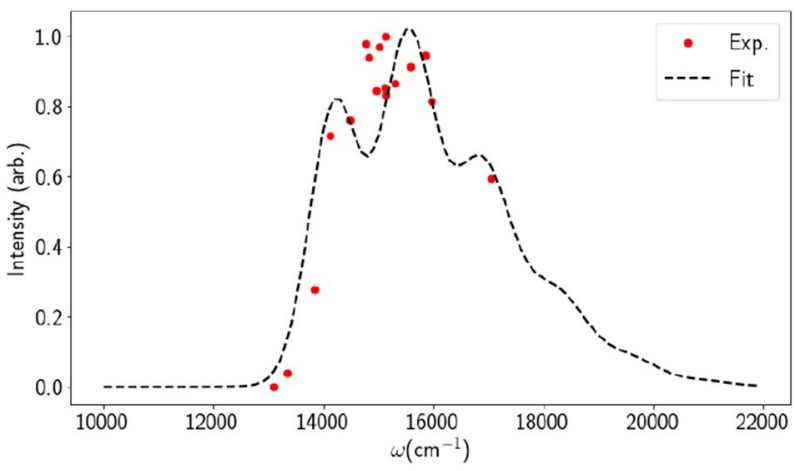

FIGURE 1 | (A) Calculated linear absorption spectrum of LHCll derived from one of the minima (red line) compared to the experimental spectrum (dashed line) (Krüger et al., 2010). The second derivative of the calculated absorption is shown (blue line) to highlight the Chl a and b peaks. The calculated spectra were essentially identical for all LHCll minima probed. (B) Calculated and experimental fluorescence profiles. (C) The calculated (dashed line) and measured linear absorption spectra of Lut in pyridine. (D) The calculated (dashed line) and measured (red dots) (Walla et al., 2002) TPE spectrum of Lut. All calculation parameters were taken from the TA fit apart from a $150 \mathrm{~cm}^{-1}$ blue-shift to account for the fact that the TPE measurements were performed in octanol.

spheroidene Fujii et al. (1998). These have a much less defined vibronic structure and deconvolution suggests that the largest peak is the $0-2$ line $\left(\left|0_{00}\right\rangle \rightarrow\left|1_{01}\right\rangle\right.$ in our model $)$ rather than $0-1$. We found it impossible to reproduce such a lineshape while retaining any kind of fit to the TA and TPE data. This may be a limit of the displaced oscillator model but it was later suggested that $S_{1}$ FL measurements may be distorted by the presence of cis-isomers (Christensen et al., 2007).

- The $S_{1}$ lifetime, $\left\langle\tau_{S_{1} \rightarrow S_{0}}\right\rangle \approx 14 \mathrm{ps}$ : Figure $2 \mathrm{C}$ shows the simulated evolution of total population on $S_{2}, S_{1}$ and $S_{0} . S_{2} \rightarrow$ $S_{1}$ internal conversion (IC) occurs on the $\approx 100 \mathrm{fs}$ timescale while $S_{1}$ undergoes near mono-exponential decay in within the $10-20$ ps range usually quoted for xanthophylls.

- Vibrational relaxation on $S_{1},\left\langle\tau_{\mathrm{vib}-S_{1}}\right\rangle \approx 1 \mathrm{ps}$ : Figure 2D shows the simulated population evolution of the $S_{1}$ vibronic levels $\left|1_{00}\right\rangle,\left|1_{10}\right\rangle$ and $\left|1_{01}\right\rangle$. While it is difficult to assign a single lifetime to a multi-component process it is clear that vibrational relaxation on $S_{1}$ is an order of magnitude faster than $S_{1}$ IC but far from instantaneous.

- Vibrational relaxation on $S_{0},\left\langle\tau_{\mathrm{vib}-S_{0}}\right\rangle<14 \mathrm{ps}$ : There is a very small transient population on $\left|0_{01}\right\rangle$ and $\left|0_{10}\right\rangle$ which reaches a peak at $\approx 9$ ps (not shown) and makes a very small contribution to the blue shoulder on the $S_{1}$ ESA. However, unlike Carts such as canthaxanthin and rhodoxanthin (Balevicius et al., 2019), the $S_{1}$ IC is too slow to generate a vibrational population inversion on $S_{0}$ and hence there is no $S^{*}$-type signal (Balevicius et al., 2019).

\subsection{Excitonic States and Intermolecular Couplings}

As a baseline we first simulated relaxation in the LHCII crystal structure (Liu et al., 2004) following protonation and minimization. The Chl-Chl couplings are essentially as previously reported (Novoderezhkin et al., 2004; Müh et al., 2010) and diagonalization leads to a set of exciton states that have already been described elsewhere (Novoderezhkin et al., 2004). Briefly, in the range 15,200 to $15,500 \mathrm{~cm}^{-1}$ we find a set of almost single-molecule (unmixed) Chl $b$ states. Between 14,700 and $15,200 \mathrm{~cm}^{-1}$ are a set of excitonic states typically localized on dimers or trimers of $\mathrm{Chl} a$. Particularly relevant to NPQ is the terminal emitter state at around $14,730 \mathrm{~cm}^{-1}$ which 

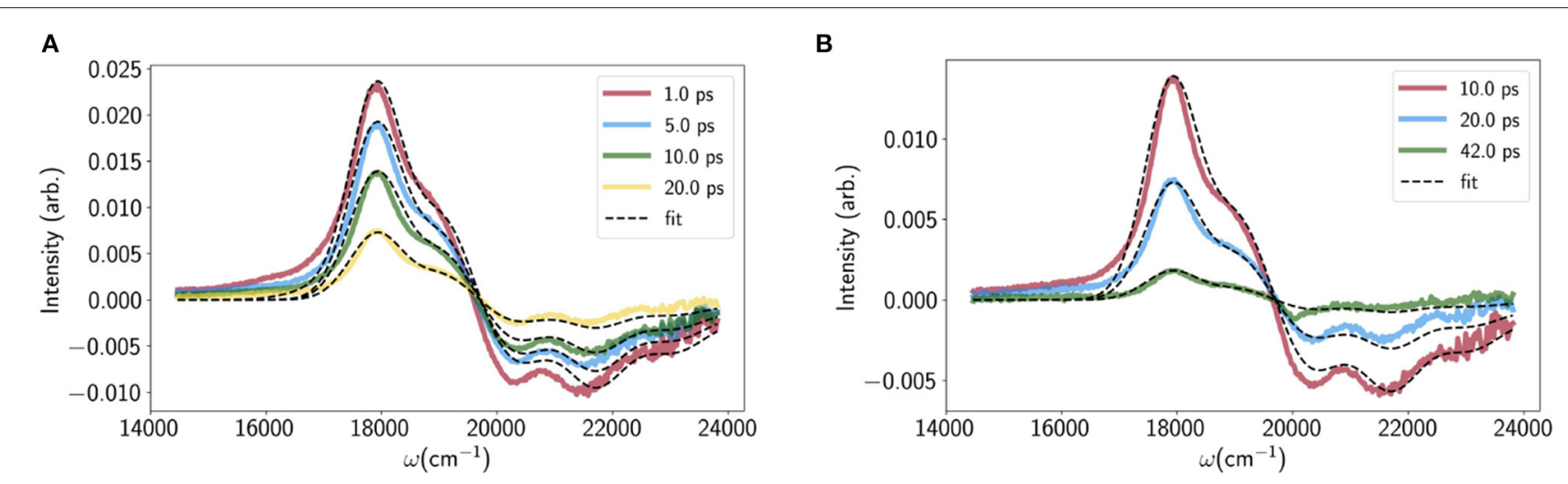

C

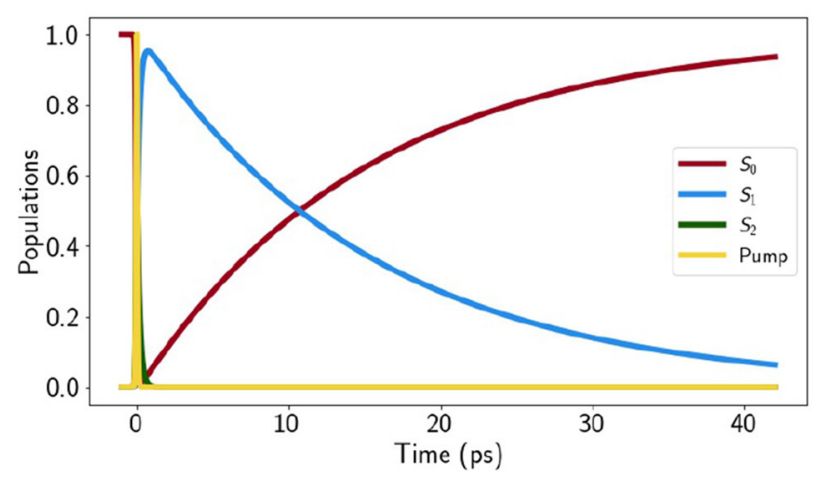

D

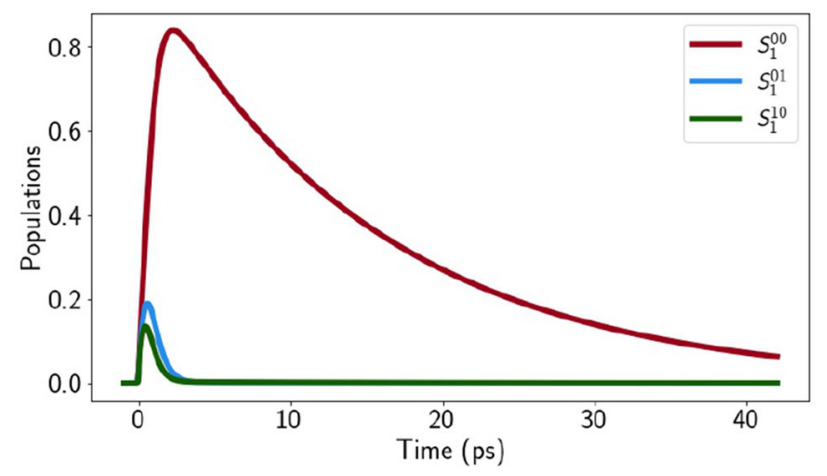

FIGURE 2 | Transient absorption traces for (A) medium times and (B) long times, with experimental data shown in solid, colored lines and model fits shown as dashed lines. (C) The evolution of the total population on each electronic state as a function of time, along with the temporal shape of the pump pulse. Note that a large part of the $S_{2} \rightarrow S_{1}$ decay (green line) overlaps with the pump. (D) Population evolution of the 3 lowest vibrational levels on $S_{1}$.

is localized on the Chl a610-a611-a612 domain closely associated with Lut1, which we label $\left|\mathrm{TE}^{-}\right\rangle$. There is also an analogous 'anti-bonding' state, $\left|\mathrm{TE}^{+}\right\rangle$, at around $15,120 \mathrm{~cm}^{-1}$. This is shown diagrammatically in Figure 3.

We then consider the purely electronic Chl-Lut1 couplings, $J_{n, L u t}^{0}$ using Lut transition charges from Khokhlov and Belov (2019). In the site basis we have the same picture as previously reported (Chmeliov et al., 2015), weak (10 $20 \mathrm{~cm}^{-1}$ ) couplings to the terminal emitter Chls and negligible couplings otherwise. Excitonic mixing among the Chls naturally mixes these couplings, the strongest $\left(\left|J_{i, L u t}^{0}\right| \approx 7 \mathrm{~cm}^{-1}\right)$ being between Lut1 and $\left|\mathrm{TE}^{-}\right\rangle$and $\left|\mathrm{TE}^{+}\right\rangle$. These small couplings justify (Balevicius and Duffy, 2020) our mixed kinetic model in which the Chl excitonic and Lut1 vibronic subsystems can exchange energy incoherently. When we model the relaxation kinetics the presence of Lut1 results in a decreased excitation lifetime of $\tau_{\mathrm{ex}} \approx 500 \mathrm{ps}$, compared to the unquenched value of $4 \mathrm{~ns}$ (Pascal et al., 2005). The pathway is two-fold, involving fairly-reversible transfer from $\left|\mathrm{TE}^{+}\right\rangle$to the nearresonant $\left|S_{1}^{10}\right\rangle=\left|1_{10}\right\rangle$ level and steep down-hill transfer from $\left|\mathrm{TE}^{-}\right\rangle$to $\left|S_{1}^{00}\right\rangle=\left|1_{00}\right\rangle$ (see Figure 4A). Recent femtosecond stimulated Raman spectroscopy (Artes Vivancos et al., 2020) has also suggested that vibrationally excited states on Lut 1 can participate in light-harvesting by internal conversion from S2 and then energy transfer to the chlorophylls, which agrees well with our observation that the $\left|1_{10}\right\rangle$ state is resonant with $|\mathrm{TE}+\rangle$. The transfer is typically slow, however. For example, the rate constant for transfer from $\left|\mathrm{TE}^{-}\right\rangle$to $\left|S_{1}^{00}\right\rangle$ is $k_{S_{1}^{00}, \mathrm{TE}^{-}}^{-1} \approx 300 \mathrm{ps}$. There are, however, several pathways that contribute, involving other exciton states and the $\left|S_{1}^{10}\right\rangle$ vibronic level, resulting is a net timescale of $\approx 100$ ps. This is too slow for any transient accumulation of population on $\left|S_{1}^{00}\right\rangle$ (see Figure 4B).

While these results are essentially identical to those previously reported (Chmeliov et al., 2015), it is important to realize that the absolute value of $\tau_{\mathrm{ex}}$ may not be quantitatively accurate, due to the fact that the Chl-Lut couplings are derived from unscaled $S_{1}$ transition charges from quantum chemical calculations (Khokhlov and Belov, 2019), but the relative changes in lifetime between minima are meaningful.

\subsection{Exploring the LHCII Potential Energy Surface}

We calculated the average, relative mean excitation times for several minima identified by a previous steered MD study (Daskalakis et al., 2020). It was reported that different monomers within the same LHCII trimer could access different 


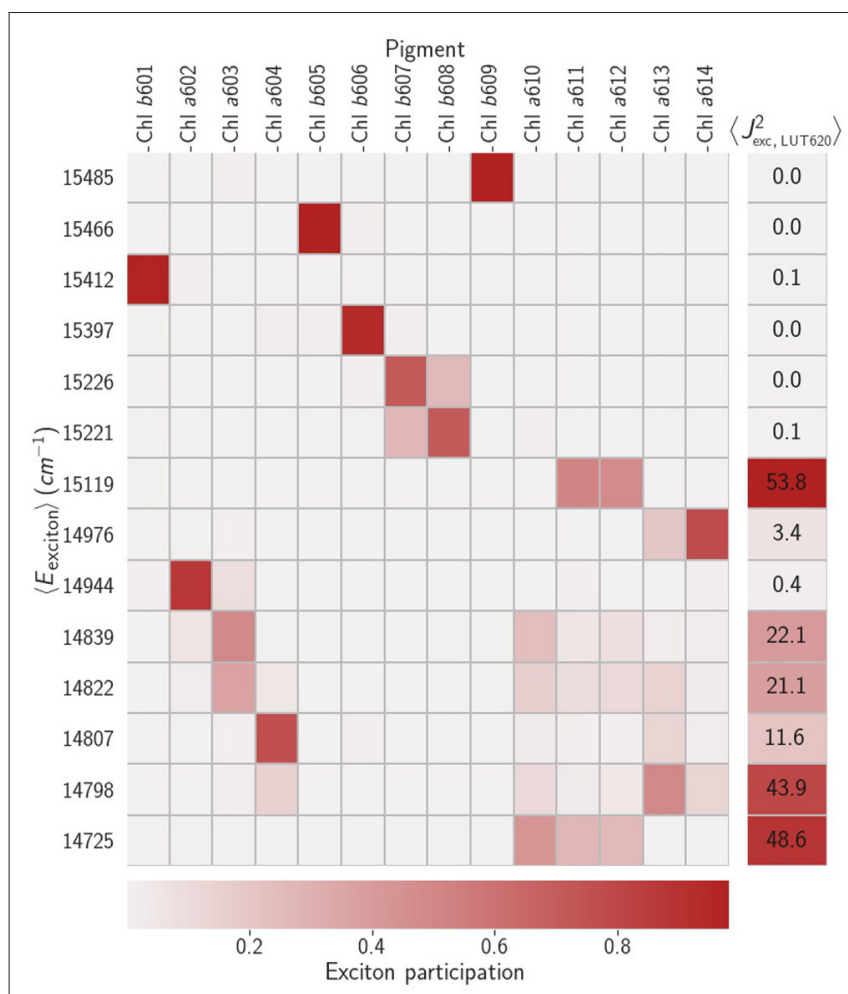

FIGURE 3 | The grid shows the average energies, $\left\langle E_{i}\right\rangle$, of the excitonic states and the average exciton participations, $\left\langle\left|c_{i}^{n}\left(t_{k}\right)\right|^{2}\right\rangle$, for a typical minimum. The right-most column lists typical average values of the square couplings between the exciton states and the $0-0$ transition on Lut. The coupling to higher vibronic transitions are simply weighted by the relevant Franck-Condon overlaps.

conformational states and so we consider the monomer in our calculations. The minima are broadly classified into 'low $\mathrm{pH}$ ' and 'neutral $\mathrm{pH}$ ' depending on the protonation state of several lumen-exposed residues. In all minima there were fluctuations in the snapshot lifetimes, $300<\tau_{\text {ex }}<1000 \mathrm{ps}$, but the average value varies little within the range $500<\left\langle\tau_{e x}\right\rangle<600$ ps (see Figures 4C,D).

It is premature to say that 'all of these minima are quenched' but we can state that there is no evidence of a simple, purelygeometric switch between states with significantly different lifetimes. We find (as previously noted Fox et al., 2017) that $\tau_{\mathrm{ex}}$ is correlated with Lut1-Chl $a 612$ coupling but the coupling is not sufficiently sensitive to the small movements of the pigments to produce any kind of functional transition.

\section{5. $S_{1}$ Excitation Energy and Asymmetry Between Lut1 and Lut2}

If we trust the TA-derived value of $\varepsilon_{S_{1}}=14,050 \mathrm{~cm}^{-1}$ then the relative arrangement of excitonic and vibronic levels is notable (see Figure 4A). $\left|T E^{+}\right\rangle$and $\left|S_{1}^{10}\right\rangle$ are near-resonant but since $\left|T E^{+}\right\rangle$acquires little exciton population at room temperature (see Figure 4B) this is not a very effective pathway for quenching. The terminal emitter state, $\left|T E^{-}\right\rangle$, lies almost precisely in the middle of $\left|S_{1}^{00}\right\rangle$ and $\left|S_{1}^{10}\right\rangle$ meaning any reasonable shift in the relative energy actually increases the quenching. This is shown in Figure 5A where we alter $\varepsilon_{S_{1}}$ to bring either $\left|S_{1}^{00}\right\rangle\left(\varepsilon_{S_{1}}=\right.$ $\left.14,750 \mathrm{~cm}^{-1}\right)$ or $\left|S_{1}^{10}\right\rangle\left(\varepsilon_{S_{1}}=13,600 \mathrm{~cm}^{-1}\right)$ into resonance with $\left|T E^{-}\right\rangle$. In both cases $\left\langle\tau_{\mathrm{ex}}\right\rangle$ drops by around $50 \%$ to roughly 300 ps. Within the smaller range we find that changes in the energy of $\varepsilon_{S_{1}}=14,050 \pm 300 \mathrm{~cm}^{-1}$, i.e., within the error bar of the reported value, the largest decrease is by about $25 \%$. For $\varepsilon_{S_{1}}>18,000 \mathrm{~cm}^{-1}$ the quenching disappears completely $\left(\left\langle\tau_{\mathrm{ex}}\right\rangle \rightarrow \Gamma_{\mathrm{Chl}}=4 \mathrm{~ns}\right)$, as has been previously reported (Lapillo et al., 2020). This is simply because there is no energetic overlap between the two sub-systems and energy transfer between them is impossible by construction.

Although we initially excluded Lut 2 we then put it back into the model, assuming the same transition charges and, initially, the same excitation energy as Lut1. The binding pocket of Lut2 (superficially) mirrors that of Lut1 with weak couplings to Chls a603 and a604 which participate in several excitonic states between $\left|T E^{-}\right\rangle$and $\left|T E^{+}\right\rangle$. This leads to a $40 \%$ decrease in lifetime relative to the Lut1-only model. Figure 5B shows the excitation population on the Lut ground state at time $t=\tau_{\mathrm{ex}}$ and we see that when $\varepsilon_{S_{1}}^{\text {Lut2 }}=\varepsilon_{S_{1}}^{\text {Lut1 }}=14,050 \mathrm{~cm}^{-1}$ Lut2 is almost as effective a quencher as Lut1. This is contrary to the observed features of NPQ and the known properties of Lut2. The initial TA measurements that lead to the proposal of a Lut-mediated NPQ identified Lut1 as the sole quencher (Ruban et al., 2007). This is likely because Lut 2 has a significantly distorted electronic structure relative to that in solution. The $S_{2}$ excitation energy of Lut 2, $\varepsilon_{S_{2}}^{\text {Lut2 }}$, is significantly lower than $\varepsilon_{S_{2}}^{\text {Lut1 }}$ (Son et al., 2020) and if this shift is caused by twisting of the backbone then it is likely accompanied by a concomitant upward-shift in $\varepsilon_{S_{1}}^{\text {Lutl }}$ (Wei et al., 2019; Artes Vivancos et al., 2020). In fact, recent ultra-broadband 2D measurements on LHCII identified a dark state (termed $S_{X}$ ), lying above the Chl a $Q_{y}$ band, which belongs exclusively to Lut2 (Son et al., 2019). This is most likely a strongly-distorted $S_{1}$. Figure $5 \mathbf{B}$ shows that quenching by Lut 2 can be completely abolished if we introduce some energetic asymmetry between Lut1 and Lut2. The shifts are not actually that large with $\varepsilon_{S_{1}}^{\text {Lut1 }}=$ $13,600 \mathrm{~cm}^{-1}$ being almost within the error bars of the measured value of $14,050 \pm 300 \mathrm{~cm}^{-1}$ and $\varepsilon_{S_{1}}^{\text {Lut2 }}=15,000-15,500 \mathrm{~cm}^{-1}$ being roughly in the region of the proposed $S_{X}$ state. It is important to note that this is not a rigorous analysis, which would require independent, in situ parameterization of Lut1 and Lut2. However, it points to an energetic sensitivity in the quenching pathway(s) that was absent in previous models.

\section{DISCUSSION}

The essence of the quenching mechanism investigated here (and previously proposed Balevičius et al., 2017) is that trivial geometric modulation of Chl-Lut coupling is sufficient to drive the system between quenched and unquenched states. This appears to be incorrect, as the complex simply does not seem to possess the conformational flexibility to induce significant changes in the coupling. We are not saying that the different minima do not represent different functional 


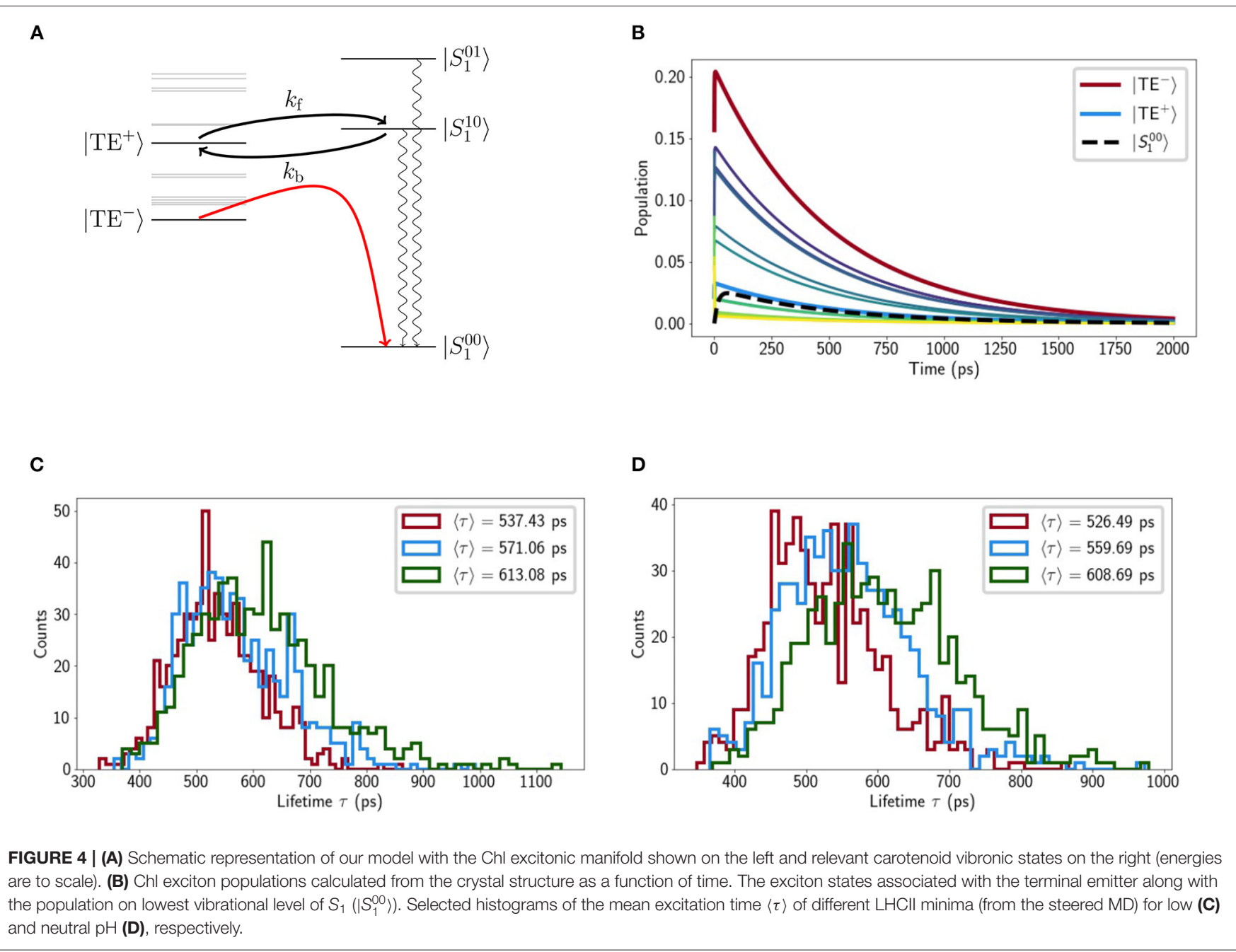

states or that carotenoids are not involved in quenching, merely that our model does not capture its key features. There are several possible quenching scenarios that can be discussed.

\subsection{NPQ May Involve Modulation of the Properties of $S_{1}$}

The point of this study was to try and cast this problem in terms of experimental parameters, specifically the $S_{1}$ energy, vibronic structure and relaxation dynamics. While the TA fits seem reliable, the data is for Lut in pyridine and obviously there is a question of whether this can be applied to Lut in protein. Actually, the default value of $\varepsilon_{S_{1}}=14,050 \mathrm{~cm}^{-1}$ was taken from NIR measurements on Lut in LHCII, although the error bars are quite big $\left( \pm 300 \mathrm{~cm}^{-1}\right)$ and the study did not compare quenched and unquenched configurations (Polívka et al., 2002). An earlier NPQ model proposed that quenching was induced by bringing the Chl $Q_{y}$ band and $S_{1}$ into resonance (Holleboom and Walla, 2014), which would require either $\varepsilon_{S_{1}}^{00}$ or $\varepsilon_{S_{1}}^{10} \approx$ $15,000 \mathrm{~cm}^{-1}$. Balevičius Jr. and Duffy recently provided a very general physical argument as to why fine tuning of this energy gap cannot modulate quenching (Balevicius and Duffy, 2020) and showed that significant quenching is possible for large energy gaps, even if the quenching state lies above the donor state. $S_{1}$ has to be quite far above the $Q_{y}$ band to abolish quenching, as was recently proposed by Lapillo et al. (2020). They reported a sharp dependence of the EET rate (and overall quenching) on the energy gap when $\varepsilon_{S_{1}} \approx 18,000 \mathrm{~cm}^{-1}$. This is simply because at this point the $Q_{y}$ band coincides with the steep red edge of the $S_{1}$ lineshape. However, as they point out, $\varepsilon_{S_{1}}$ is not a free parameter and a protein-induced blue-shift of $14,050 \rightarrow 18,000 \mathrm{~cm}^{-1}(712 \rightarrow 555 \mathrm{~nm})$ would be quite large. Saccon et al. recently performed TA measurements on quenched LHCII immobilized in polyacrylamide gel (a model for NPQ) (Saccon et al., 2020) and found that linear excitation of Lut (i.e., via $S_{2}$ ) produces the usual $S_{1} \rightarrow S_{n}$ ESA at $\varepsilon_{S_{n}}-\varepsilon_{S_{1}} \approx$ $18,500 \mathrm{~cm}^{-1}(540 \mathrm{~nm})$. This is reasonably close to the value in pyridine, $\varepsilon_{S_{n}}-\varepsilon_{S_{1}} \approx 17,900 \mathrm{~cm}^{-1}(558 \mathrm{~nm})$. Of course this is an indirect measurement and a massive shift in $\varepsilon_{S_{1}}$ could be hidden by a correlated shift in $\varepsilon_{S_{n}}$. However, this would have a significant affect on the ESA formation and decay kinetics which is not observed. 


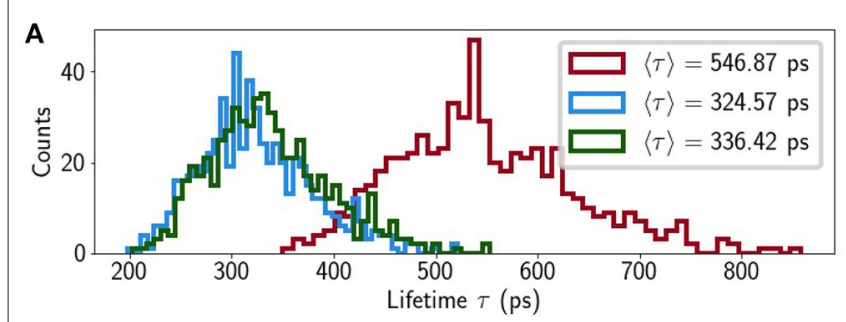

B

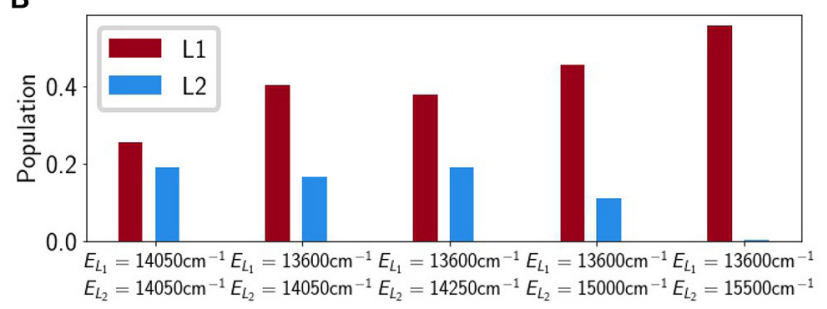

FIGURE 5 | (A) Lifetime histograms with $E_{S_{1}}$ set to $14,050 \mathrm{~cm}^{-1}$ (red), 13,600 $\mathrm{cm}^{-1}$ (blue) and $14,750 \mathrm{~cm}^{-1}$ (green), and (B) average populations on $\mathrm{L} 1$ and $L 2$ at $\tau$, where $E_{L_{i}}$ denotes the $S_{1}^{00}$ energy assigned to Lut $i$.

\subsection{NPQ May Involve Non-coulomb Interactions and/or Non-adiabatic Inter-molecular States}

When estimating Chl-Chl couplings the $Q_{y}$ transition density/charges are typically re-scaled to reproduce an experimentally determined oscillator strength (Knox and Spring, 2003; Müh et al., 2010). This is clearly not an option for $S_{1}$ (for which a non-zero oscillator strength has never been measured) and therefore the absolute values of $S_{1}$ transition charges and the Chl-Lut couplings are difficult to estimate. For the planar geometry of Lut the published transition atomic charges (Khokhlov and Belov, 2019) yield a dipole moment of $\left|\mu_{S_{1}}\right| \approx 0.1-0.2 D$ which, although very small, is nonzero $\left(\left|\mu_{Q_{y}}\right| \approx 3-5 D\right.$ for comparison Knox and Spring, 2003). It is possible that the amplitude of the $S_{1}$ transition density is overestimated and therefore so are the Coulomb couplings, $Q_{y} \rightarrow S_{1}$ transfer rates, and overall level of quenching. In fact, given that there appears to be insufficient conformational flexibility in LHCII to switch this Coulomb-mediated quenching off, it may be an artifact. The reason that it was initially considered promising was that it qualitatively matched the NPQ scheme proposed by Ruban et al. in 2007, based on TA measurements of quenched LHCII aggregates (Ruban et al., 2007). The role of $S_{1}$ was implied by global target analysis of the kinetics rather than any visually detectable $S_{1}$ signal and so must be treated with caution. Direct observation of $S_{1}$-mediated quenching was later reported for the cyanobacterial High light inducible proteins (Hlips) which are ancestors of LHCII (Staleva et al., 2015). Hlips are perpetually quenched by $\approx 2$ ps (hence observable) EET from a small pool of $\mathrm{Chl}$ a to $\beta$-carotene in one of the central binding pockets that are analogous to L1 and L2 in LHCII. More recently, sub-picosecond EET to Lut1 was directly observed in LHCII via ultra-fast 2D spectroscopy (Son et al., 2020). In both the EET is much faster than predicted by this or any of the previous models and it is difficult to see how such fast transfer could be Coulomb-mediated and be in any way switchable or involve an optically-forbidden transition. Cignoni et al. provide a possible answer via a detailed QM/MM study of CP29 in which short-range interactions (exchange, overlap, etc.) were found to make large contributions to the Chl-Cart couplings (Cignoni et al., 2021). These are naturally far more sensitive to minor conformational changes than the long-range Coulomb interactions.

The picture gets even more complicated when one considers quenched LHCII in gel. It was recently shown that excitation of $Q_{y}$ results in the immediate appearance of a large-amplitude positive peak at $19,417 \mathrm{~cm}^{-1}(515 \mathrm{~nm})$ which we'll label $A_{515}$ (Saccon et al., 2020). This is not merely a shifted $S_{1}$ as direct excitation of Lut gave the usual $S_{1} \rightarrow S_{n}$ ESA at $18,500 \mathrm{~cm}^{-1}$ (540 nm), although $A_{515}$ is detectable at later times and may simply be initially hidden by $S_{1}$. This suggests that $S_{2} \rightarrow S_{1}$ and $S_{2} \rightarrow A_{515}$ are competing pathways. $A_{515}$ is in the region of the $S^{*}$ signal which some people suggest is either a distorted $S_{1}$ or a dipole-forbidden singlet electronic state lying below $S_{1}$ (Mascoli et al., 2019). That argument aside, since the Chl ESA is typically flat and featureless, it seems reasonable to assume that $A_{515}$ is associated with the Cars, although it is difficult to assign it solely to Lut1. $A_{515}$ is independent of whether it is Chl $a$ or Chl $b$ that is excited and the GSB bands in the $S_{2}$ region $(<500$ $\mathrm{nm}$ ) looks very different to the classic $S_{2}$ GSB. This all suggests some type of delocalized quenching pathway that involves several Carts and possibly even some non-adiabatic intermolecular states not accessible simply by exciting $S_{2}$. This is exactly the picture emerging from the elaborate QM/MM models of CP29 being reported by Mennucci et al. (Cupellini et al., 2020; Lapillo et al., 2020).

\subsection{Quenching Requires Hydrophobic Mismatch and Aggregation}

It is possible that the conformational switch cannot be revealed by simulating a single LHCII monomer/trimer in a lipid bilayer. In vitro quenching is induced by low detergent concentration which in solution leads to aggregation. LHCII aggregates are the original model system for studying NPQ (Horton et al., 1991) and there is compelling evidence that some form of aggregation or clustering in the membrane is part of the in vivo mechanism (Johnson et al., 2011). Key to this is PsbS, with overexpression observed to enhance LHCII clustering and its absence frustrating it (Goral et al., 2012). Recent simulations show that PsbS's lumen-exposed side is covered in titratable residues with protonation causing an unfolding of a specific region implicated in protein-protein interactions (Liguori et al., 2019). Other studies have shown that it is capable of interacting with the minor PSII antenna complexes (Daskalakis, 2018), possibly helping LHCII to partially detach from the reaction center complex and form the quenching clusters. It has also been suggested that active PsbS has an affinity for certain lipids, altering local membrane composition and causing the hydrophobic mismatch 
that drives aggregation/clustering (Daskalakis et al., 2019; Ruban and Wilson, 2020).

The role of external interactions on LHCII conformation was (at least partially) considered in the metadynamical simulations used in this work (Daskalakis et al., 2020). They were performed by first calculating the free energy surface (FES) of LHCII while considering a wide range of external stimuli, such as $\Delta \mathrm{pH}$ and interactions with PsbS. The next step involved steering MD simulations of an LHCII trimer around this FES in order to reach the minima. Equilibrium MD trajectories were then performed on an isolated trimer once each minimum was reached. Due to the absence of external stimuli in these equilibrium trajectories, it is possible that the configuration space even at these minima still favors the quenched conformation over the unquenched.

\section{METHODS}

\subsection{TA Measurements of Lutein in Pyridine}

All transient absorption data were measured with a spectrometer described in detail in Saccon et al. (2020). Lutein (Sigma Aldrich) was dissolved in spectroscopic grade pyridine to yield an optical density of $\approx 0.2 \mathrm{~mm}^{-1}$ at the absorption maximum. The sample was placed in a $2 \mathrm{~mm}$ path-length quartz cuvette equipped with a micro-stirrer to avoid sample degradation during measurement. The mutual polarization of the excitation and probe beams was set to the magic angle $\left(54.7^{\circ}\right)$ and excitation intensity was kept below 1014 photons pulse $\mathrm{cm}^{-1}$.

\subsection{The Chlorophyll Exciton Manifold}

Modeling of energy relaxation within the chlorophylls is carried out according to previous work (Novoderezhkin et al., 2004; Malý et al., 2016) and is described in detail in section B of the Supporting Information. Briefly, for a single uncorrelated MD snapshot (at time $t_{k}$ ) the relevant system of Chl excited $\left(Q_{y}\right)$ states is determined by the usual spin-boson Hamiltonian,

$$
\mathcal{H}\left(t_{k}\right)=\sum_{n} E_{n}|n\rangle\left\langle n\left|+\sum_{m \neq n} J_{m n}\left(t_{k}\right)\right| m\right\rangle\langle n|
$$

where $\{|m\rangle\}$ is the 'site' basis of uncoupled single-molecule excitations, $\left\{E_{m}\right\}$ are the site (excitation) energies and $\left\{J_{m n}\left(t_{k}\right)\right\}$ are the resonance couplings. $\left\{J_{m n}\left(t_{k}\right)\right\}$ are calculated as the sum of pairwise Coulomb interactions between transition atomic charges, $\left\{q_{\alpha}\right\}$,

$$
J_{m n}\left(t_{k}\right)=\frac{1}{4 \pi \varepsilon} \sum_{\substack{\alpha \in m \\ \beta \in n}} \frac{q_{\alpha} q_{\beta}}{\left|\mathbf{r}_{\alpha}\left(t_{k}\right)-\mathbf{r}_{\beta}\left(t_{k}\right)\right|}
$$

where $\varepsilon=\varepsilon_{r} \varepsilon_{0}=2 \varepsilon_{0}$. Both $\left\{E_{n}\right\}$ and $q_{\alpha}$ are taken from Müh et al. (Madjet et al., 2006; Renger et al., 2007). Equation (1) is then diagonalised to give the exciton basis,

$$
|i\rangle=\sum_{n} c_{n}^{i}|n\rangle
$$

where $c_{n}^{i}$ are the participation coefficients of each pigment state, $|n\rangle$ for a given exicton state, $|i\rangle$. Site energies, oscillator strengths and couplings to Cart vibronic levels are also mixed. The exciton states are initially populated according to their oscillator strengths and relaxation is modeled using the approach outlined in Novoderezhkin et al. (2004). The population relaxation rates are given by,

$$
k_{i j}=\sum_{n}\left|c_{n}^{i}\right|^{2}\left|c_{n}^{j}\right|^{2}\left(1+\operatorname{coth}\left(\frac{\hbar \omega_{i j}}{2 k_{B} T}\right)\right) C_{n}^{\prime \prime}\left(\omega_{i j}\right)
$$

where $C_{n}^{\prime \prime}(\omega)$ is the spectral density of bath-induced site energy fluctuations and $\omega_{i j}$ is the gap between the zero-phonon lines of excitons $i$ and $j$. The ansatz spectral density (Novoderezhkin et al., 2004) is assumed throughout. For a single uncorrelated snapshot along a trajectory (at time $t_{k}$ ) the instantaneous LA and FL spectra are given by

$$
A\left(\omega ; t_{k}\right) \propto \omega \sum_{i} \chi_{i}\left(\omega ; t_{k}\right)
$$

and,

$$
F\left(\omega ; t_{k}\right) \propto \omega^{3} \sum_{i} \tilde{\chi}_{i}\left(\omega ; t_{k}\right) P_{i}\left(\infty ; t_{k}\right)
$$

where $\left\{\chi_{i}\left(\omega, t_{k}\right)\right\}$ and $\left\{\tilde{\chi}_{i}\left(\omega ; t_{k}\right)\right\}$ are the instantaneous LA and FL line-shapes, respectively and $\left\{P_{i}\left(\infty ; t_{k}\right)\right\}$ are the steady state populations of the exciton states. The true LA and FL for a particular minima are given by averaging over a trajectory, $\left\{J_{m n}\left(t_{k}\right)\right\}$, and then again over several instances of Gaussian disorder in the Chl site energies, $\left\{E_{m}\right\}$.

\subsection{The Carotenoid Vibronic Subsystem}

The full VERA (Balevičius et al., 2018; Balevicius et al., 2019) Hamiltonian is,

$$
\begin{aligned}
\mathcal{H}_{\mathrm{car}} & =\mathcal{H}_{\mathrm{S}}+\mathcal{H}_{\mathrm{B}}+\mathcal{H}_{\mathrm{SB}}^{\mathrm{IVR}}+\mathcal{H}_{\mathrm{SB}}^{\mathrm{IC}} \\
& =\sum_{\substack{i \\
a_{1} \\
a_{2}}} \varepsilon_{i}^{a_{1} a_{2}}\left|i_{a_{1}, a_{2}}\right\rangle\left\langle i_{a_{1}, a_{2}}\right| \\
& +\sum_{\kappa}\left(\frac{p_{\kappa}^{2}}{2 m_{\kappa}}+\frac{m_{\kappa} \omega_{\kappa}^{2} x_{\kappa}^{2}}{2}\right) \\
+ & {\left[\sum_{i, \kappa} c_{i \kappa} x_{\kappa} \sqrt{a_{1}+1}\left(\left|i_{a_{1}, a_{2}}\right\rangle\left\langle i_{a_{1}+1, a_{2}}|+| i_{a_{1}+1, a_{2}}\right\rangle\left\langle i_{a_{1}, a_{2}}\right|\right)\right.} \\
+ & \left.\sum_{\substack{i, \kappa \\
c_{i \kappa}}} x_{\kappa} \sqrt{a_{2}+1}\left(\left|i_{a_{1}, a_{2}}\right\rangle\left\langle i_{a_{1}, a_{2}+1}|+| i_{a_{1}, a_{2}+1}\right\rangle\left\langle i_{a_{1}, a_{2}}\right|\right)\right] \\
+ & {\left[\sum_{\substack{i, \kappa \\
a_{1}, a_{2} \\
b_{1}, b_{2}}} f_{i \kappa} x_{\kappa}\left(\prod_{\alpha=1,2} F_{i_{a_{\alpha}}, i+1_{b_{\alpha}}}^{\alpha}\right)\right.} \\
+ & \sum_{\substack{i, \kappa \\
a_{1}, a_{2} \\
b_{1}, b_{2}}} f_{i \kappa} x_{\kappa}\left(\prod_{\alpha=1,2} F_{i+1_{a_{\alpha},}, i_{b_{\alpha}}}^{\alpha}\right)\left|i+i_{a_{1} a_{2}}\right\rangle\left\langle i+1_{b_{1} b_{2}}\right|
\end{aligned}
$$


Equation (7b) is the Hamiltonian of the system $\left(\mathcal{H}_{\mathrm{S}}\right)$ of uncoupled vibronic levels, $\left\{\left|i_{a_{1} a_{2}}\right\rangle\right\}$, where

$$
\varepsilon_{i}^{a_{1} a_{2}}=\epsilon_{i}+\epsilon_{a_{1}, a_{2}}=\epsilon_{i}+\left(a_{1}+\frac{1}{2}\right) \hbar \omega_{1}+\left(a_{2}+\frac{1}{2}\right) \hbar \omega_{2}
$$

is the sum of the electronic, $\epsilon_{i}$ and vibrational energies. $\mathcal{H}_{\mathrm{B}}$ is the bath Hamiltonian which is composed of a large set of harmonic oscillators representing the non-optical modes of the Cart itself plus librations, solvent modes, etc. We split this into two parts, $\mathcal{H}_{\mathrm{SB}}^{\mathrm{IVR}}$ and $\mathcal{H}_{\mathrm{SB}}^{\mathrm{IC}}$. $\mathcal{H}_{\mathrm{SB}}^{\mathrm{IVR}}$ describes the bath-induced couplings between adjacent vibrational levels of the optical modes and are therefore responsible for vibrational relaxation on the electronic states. $\left\{c_{i \kappa}\right\}$ are the coupling constants and $\left\{x_{\kappa}\right.$ the bath mode displacements. Energy (mostly) relaxes into the non-optical modes of the Cart and therefore reflects Intramolecular Vibrational Redistribution (IVR). Note that there is no population transfer between the two optically-coupled modes. $\mathcal{H}_{\mathrm{SB}}^{\mathrm{IC}}$ couples different electronic states and is therefore responsible for Internal Conversion (IC). It is characterized by coupling constants, $f_{i \kappa}$, and the Franck-Condon (FC) overlaps,

$$
F_{i_{a_{\alpha}}, j_{b_{\alpha}}}^{\alpha}={ }^{i}\left\langle a_{\alpha} \mid b_{\alpha}\right\rangle^{j}
$$

If we assume that the frequencies of the optical modes $\left(\omega_{\alpha}\right)$ are independent of the electronic state (i.e., no Duschinsky rotations) then $\left\{F_{i_{a_{\alpha}}, j_{b_{\alpha}}}^{\alpha}\right\}$ are entirely determine by their relative dimensionless displacements, $\left\{d_{\alpha}^{i j}\right\}$. The relaxation dynamics are obtained by a second-order perturbative treatment of $\mathcal{H}_{\mathrm{SB}}^{\mathrm{IVR}}$ and $\mathcal{H}_{\mathrm{SB}}^{\mathrm{IC}}$. The resulting equations of motion are rather complicated and are listed in section $\mathrm{C}$ of the Supporting Information. The various IVR, $k_{\alpha}^{ \pm}$, and IC, $k_{a_{1} a_{2}, b_{1} b_{2}}^{i j}$, rate constants are defined in terms of Drude-type spectral density functions $C_{i_{\alpha}}^{\prime \prime}(\omega)$ and $C_{i_{\alpha}, j_{\alpha}}^{\prime \prime}(\omega)$. We therefore have a large set of fitting parameters including electronic, $\left\{\epsilon_{i}\right\}$ and vibrational, $\epsilon_{a_{1} a_{2}}$, energies, modes frequencies, $\omega_{\alpha}$, mode displacements, $d_{\alpha}^{i j}$, and the reorganization energies, $\lambda_{i_{\alpha}}, \lambda_{i_{\alpha}, j_{\alpha}}$, and dephasing frequencies, $\gamma_{i_{\alpha}}, \gamma_{i_{\alpha}, j_{\alpha}}$. Solving the dynamics yields a set of vibronic populations, $n_{a_{1} a_{2}}^{i}(t)$, which are used to calculation the TA difference spectrum as a combination of ESA, GSB and stimulated emission (SE) components,

$$
\Delta A(\omega, t)=A_{\mathrm{ESA}}(\omega, t)-A_{\mathrm{SE}}(\omega, t)-A_{\mathrm{GSB}}(\omega, t)
$$

which are given by,

$$
A_{X}(\omega, t)=\sum_{i, a_{1}, a_{2}} n_{a_{1} a_{2}}^{i}(t) I_{a_{1} a_{2}, X}(\omega)
$$

where $I_{a_{1} a_{2}, X}(\omega)$ are FC-weighted Gaussian/Lorentzian lineshape functions that account for line-broadening.

\subsection{Energy Transfer Between the Chlorophyll and Lutein Subsystems}

Having parameterized the subsystems separately we can now couple them via the calculated resonance couplings, $J_{n, \text { Lut }}^{0}\left(t_{k}\right)$. We make two assumptions. Firstly, since the inter-pigment couplings between the Chls and Lut is an order of magnitude smaller than the nearest-neighbor chlorophyll couplings (there is essentially no coupling between Lut1 and Lut2), we treat the Chl-Lut system as two weakly-interacting subsystems and assume that energy transfer proceeds incoherently (Balevicius and Duffy, 2020). Secondly, since there is almost no accumulation of vibronic population on the ground state ('hot' ground state), we do not explicitly include the Chl or Lut ground states in the dynamics. $S_{1}$ can decay to higher vibrational levels on $S_{0}$ but excitation proceeds from $\left|S_{0}^{00}\right\rangle=\left|0_{00}\right\rangle$. Essentially, we are assuming instantaneous IVR on the ground state. The couplings in the exciton basis are given by,

$$
J_{i, \mathrm{Lut}}^{0}\left(t_{k}\right)=\sum_{n=1}^{n_{\mathrm{Chl}}} c_{n}^{i} J_{n, \mathrm{Lut}}^{0}\left(t_{k}\right)
$$

where $\left\{J_{n, \text { Lut }}^{0}\left(t_{k}\right)\right\}$ are the purely electronic Chl-Lut couplings. The rate of transfer from $\mathrm{Chl}$ exciton state $|i\rangle$ to Lut vibronic level $\left|S_{1}^{a_{1} a_{2}}\right\rangle=\left|1_{b_{1} b_{2}}\right\rangle$ is given by the Fermi Golden Rule,

$$
\begin{aligned}
k_{i \rightarrow\left(0,0, b_{1}, b_{2}\right)}\left(t_{k}\right) & =2 \pi\left(\left|\prod_{\alpha=1,2} F_{0_{0_{\alpha}}, 1_{b_{\alpha}}}^{\alpha}\right|^{2}\right)\left|J_{i, \mathrm{Lut}}^{0}\left(t_{k}\right)\right|^{2} \\
& \times \int_{-\infty}^{\infty} d \omega \tilde{\chi}_{i}^{\prime}\left(\omega ; \omega_{i 0}-\lambda_{i}\right) \sigma\left(\omega ; \Delta_{b_{\alpha} 0_{\alpha}}^{10}, \Delta \omega_{10}\right)
\end{aligned}
$$

where $\Delta_{b_{\alpha} a_{\alpha}}^{i j}=\left(\epsilon_{i}+\epsilon_{b_{1}, b_{2}}\right)-\left(\epsilon_{j}+\epsilon_{a_{1}, a_{2}}\right)$ is the vibronic energy gap, $\tilde{\chi}_{i}^{\prime}$ is the normalized excitonic fluorescence lineshape and $\sigma\left(\omega ; \Delta_{b_{\alpha} 0_{\alpha}}^{10}, \Delta \omega_{10}\right)$ is the normalized vibronic Gaussian lineshape of width $\Delta \omega_{10}=1070 \mathrm{~cm}^{-1}$ determined by the TA fit. The backward rate is similarly defined and Boltzmann factors are added to uphill rates to enforce the detailed balance condition.

\section{DATA AVAILABILITY STATEMENT}

The original contributions presented in the study are included in the article/Supplementary Material, further inquiries can be directed to the corresponding author.

\section{AUTHOR CONTRIBUTIONS}

CD, CG, and TW devised the project. TP provided TA data and assisted with data fitting. CD performed TA data fitting. VD provided steered MD data. TW contributed pilot MD data for development of model. All calculations and overall model development by CG. CG and CD wrote the draft, all authors took part in editing.

\section{ACKNOWLEDGMENTS}

The authors thank Milan Durchan for assistance with transient absorption measurements. CG and CD thank the Biotechnology and Biological Sciences Research Council (BBSRC, BB/T000023/1). TW thanks the Chinese Scholarship Council for Ph.D. funding. TP thanks the Czech Science 
Foundation (19-28323X) for financial support. VD would like to thank European Regional Development Fund and the Republic of Cyprus through the Research and Innovation Foundation (POST-DOC/0916/0049) for supporting the metadynamics studies of LHCII trimers.

\section{REFERENCES}

Ahn, T. K., Avenson, T. J., Ballottari, M., Cheng, Y.-C., Niyogi, K. K., Bassi, R., et al. (2008). Architecture of a charge-transfer state regulating light harvesting in a plant antenna protein. Science 320, 794-797. doi: 10.1126/science.1154800

Andreussi, O., Knecht, S., Marian, C. M., Kongsted, J., and Mennucci, B. (1993). Carotenoids and light-harvesting: From dft/mrci to the tamm-dancoff approximation. J. Chem. Theory Comput. 11, 655-666. doi: 10.1021/ct5011246

Aro, E. M., Virgin, I., and Andersson, B. (1993). Photoinhibition of photosystem ii. inactivation, protein damage and turnover. Biochim. Biophys. Acta 1143, 113-134. doi: 10.1016/0005-2728(93)90134-2

Artes Vivancos, J. M., van Stokkum, I. H. M., Saccon, F., Hontani, Y., Kloz, M., Ruban, A., et al. (2020). Unraveling the excited-state dynamics and light-harvesting functions of xanthophylls in light-harvesting complex ii using femtosecond stimulated raman spectroscopy. J. Am. Chem. Soc. 142, 17346-17355. doi: 10.1021/jacs.0c04619

Balevičius Jr., V., Fox, K. F., Bricker, W. P., Jurinovich, S., Prandi, I. G., et al. (2017). Fine control of chlorophyll-carotenoid interactions defines the functionality of light-harvesting proteins in plants. Sci. Rep. 7, 13956. doi: 10.1038/s41598-017-13720-6

Balevičius Jr., V., Lincoln, C. N., Viola, D., Cerullo, G., Hauer, J., et al. (2018). Effects of tunable excitation in carotenoids explained by the vibrational energy relaxation approach. Photosynth. Res. 135, 55-64. doi: 10.1007/s11120-017-0423-6

Balevicius, V., and Duffy, C. D. P. (2020). Excitation quenching in chlorophyllcarotenoid antenna systems: 'coherent' or 'incoherent'. Photosynth. Res. 144, 301-315. doi: 10.1007/s11120-020-00737-8

Balevicius, V., Wei, T., Tommaso, D. D., Abramavicius, D., Hauer, J., Polívka, T., et al. (2019). The full dynamics of energy relaxation in large organic molecules: from photo-excitation to solvent heating. Chem. Sci. 10, 4792-4804. doi: 10.1039/C9SC00410F

Belgio, E., Duffy, C. D. P., and Ruban, A. V. (2013). Switching light harvesting complex ii into photoprotective state involves the lumen-facing apoprotein loop. Phys. Chem. Chem. Phys. 15, 12253-12261. doi: 10.1039/c3cp51925b

Bode, S., Quentmeier, C. C., Liao, P.-N., Hafi, N., Barros, T., Wilk, L., et al. (2009). On the regulation of photosynthesis by excitonic interactions between carotenoids and chlorophylls. Proc. Natl. Acad. Sci. U.S.A. 106, 12311-12316. doi: 10.1073/pnas.0903536106

Chmeliov, J., Bricker, W. P., Lo, C., Jouin, E., Valkunas, L., Ruban, A. V., et al. (2015). An 'all pigment'model of excitation quenching in lhcii. Phys. Chem. Chem. Phys. 17, 15857-15867. doi: 10.1039/C5CP01905B

Christensen, R. L., Galinato, M. G. I., Chu, E. F., Fujii, R., Hashimoto, H., and Frank, H. A. (2007). Symmetry control of radiative decay in linear polyenes: low barriers for isomerization in the s1 state of hexadecaheptaene. J. Am. Chem. Soc. 129, 1769-1775. doi: 10.1021/ja0609607

Cignoni, E., Lapillo, M., Cupellini, L., Acosta Gutierrez, S., Gervasio, F. L., and Mennucci, B. (2021). A different perspective for nonphotochemical quenching in plant antenna complexes. Nat. Commun. 12, 7152. doi: 10.1038/s41467-021-27526-8

Cupellini, L., Calvani, D., Jacquemin, D., and Mennucci, B. (2020). Charge transfer from the carotenoid can quench chlorophyll excitation in antenna complexes of plants. Nat. Commun. 11, 662. doi: 10.1038/s41467-020-14488-6

Daskalakis, V. (2018). Protein-protein interactions within photosystem ii under photoprotection: the synergy between cp29 minor antenna, subunit s (psbs) and zeaxanthin at all-atom resolution. Phys. Chem. Chem. Phys. 20, 11843-11855. doi: 10.1039/C8CP01226A

Daskalakis, V., Papadatos, S., and Kleinekathöfer, U. (2019). Fine tuning of the photosystem ii major antenna mobility within the thylakoid

\section{SUPPLEMENTARY MATERIAL}

The Supplementary Material for this article can be found online at: https://www.frontiersin.org/articles/10.3389/fpls.2021. 797373/full\#supplementary-material

membrane of higher plants. Biochim. Biophys. Acta 1861, 183059. doi: 10.1016/j.bbamem.2019.183059

Daskalakis, V., Papadatos, S., and Stergiannakos, T. (2020). The conformational phase space of the photoprotective switch in the major light harvesting complex II. Chem. Commun. 56, 11215-11218. doi: 10.1039/D0CC04486E

Duffy, C. D. P., Chmeliov, J., Macernis, M., Sulskus, J., Valkunas, L., and Ruban, A. V. (2013). Modeling of fluorescence quenching by lutein in the plant light-harvesting complex lhcii. J. Phys. Chem. B 117, 10974-10986. doi: $10.1021 /$ jp3110997

Fox, K. F., Balevicius, V., Chmeliov, J., Valkunas, L., Ruban, A. V., and Duffy, C. D. P. (2017). The carotenoid pathway: what is important for excitation quenching in plant antenna complexes? Phys. Chem. Chem. Phys. 19, 22957-22968. doi: 10.1039/C7CP03535G

Fox, K. F., Ünlü, C., Balevicius, V., Ramdour, B. N., Kern, C., Pan, X., et al. (2018). A possible molecular basis for photoprotection in the minor antenna proteins of plants. Biochim. Biophys. Acta 1859, 471-481. doi: 10.1016/j.bbabio.2018.03.015

Fujii, R., Onaka, K., Kuki, M., Koyama, Y., and Watanabe, Y. (1998). The 2ag- energies of all-trans-neurosporene and spheroidene as determined by fluorescence spectroscopy. Chem. Phys. Lett. 288, 847-853. doi: 10.1016/S0009-2614(98)00376-5

Goral, T. K., Johnson, M. P., Duffy, C. D. P., Brain, A. P. R., Ruban, A. V., and Mullineaux, C. W. (2012). Light-harvesting antenna composition controls the macrostructure and dynamics of thylakoid membranes in arabidopsis. Plant J. 69, 289-301. doi: 10.1111/j.1365-313X.2011.04790.x

Holleboom, C.-P., and Walla, P. J. (2014). The back and forth of energy transfer between carotenoids and chlorophylls and its role in the regulation of light harvesting. Photosynth. Res. 119, 215-221. doi: 10.1007/s11120-0139815-4

Holt, N. E., Zigmantas, D., Valkunas, L., Li, X. P., Niyogi, K. K., and Fleming, G. R. (2005). Carotenoid cation formation and the regulation of photosynthetic light harvesting. Science 307, 433-436. doi: 10.1126/science.11 05833

Horton, P., Ruban, A. V., Rees, D., Pascal, A. A., Noctor, G., and Young, A. J. (1991). Control of the light-harvesting function of chloroplast membranes by aggregation of the lhcii chlorophyll-protein complex. FEBS Lett. 292, 1-4. doi: 10.1016/0014-5793(91)80819-O

Horton, P., Ruban, A. V., and Wentworth, M. (2000). Allosteric regulation of the light harvesting system of photosystem ii. Philos. Trans. R. Soc. B 355, 1361-1370. doi: 10.1098/rstb.2000.0698

Ilioaia, C., Johnson, M. P., Liao, P. N., Pascal, A. A., van Grondelle, R., Walla, P. J., et al. (2011). Photoprotection in plants involves a change in lutein 1 binding domain in the major light-harvesting complex of photosystem ii. J. Biol. Chem. 286, 27247-27254. doi: 10.1074/jbc.M111.234617

Jahns, P., Latowski, D., and Strzalka, K. (2009). Mechanism and regulation of the violaxanthin cycle: the role of antenna proteins and membrane lipids. Biochim. Biophys. Acta 1787, 3-14. doi: 10.1016/j.bbabio.2008.09.013

Johnson, M. P., Goral, T. K., Duffy, C. D., Brain, A. P., Mullineaux, C. W., and Ruban, A. V. (2011). Photoprotective energy dissipation involves the reorganization of photosystem ii light-harvesting complexes in the grana membranes of spinach chloroplasts. Plant Cell. 23, 1468-1479. doi: 10.1105/tpc. 110.081646

Johnson, M. P., and Ruban, A. V. (2011). Restoration of rapidly reversible photoprotective energy dissipation in the absence of psbs protein by enhanced Sph. J. Biol. Chem. 286, 19973-19981. doi: 10.1074/jbc.M111.237255

Khokhlov, D., and Belov, A. (2019). Ab initio model for the chlorophylllutein exciton coupling in the lhcii complex. Biophys. Chem. 286, 16-24. doi: 10.1016/j.bpc.2019.01.001 
Knox, R. S., and Spring, B. Q. (2003). Dipole strengths in the chlorophylls. Photochem. Photobiol. 77, 497-501. doi: 10.1562/0031-8655(2003)077andlt;0497:DSITCandgt;2.0.CO;2

Krüger, T. P. J., Novoderezhkin, V. I., Ilioaia, C., and van Grondelle, R. (2010). Fluorescence spectral dynamics of single lhcii trimers. Biophys. J. 98, 3093-3101. doi: 10.1016/j.bpj.2010.03.028

Lapillo, M., Cignoni, E., Cupellini, L., and Mennucci, B. (2020). The energy transfer model of nonphotochemical quenching: Lessons from the minor CP29 antenna complex of plants. Biochim. Biophys. Acta 1861, 148282. doi: 10.1016/j.bbabio.2020.148282

Li, X., Gilmore, A. M., Caffarri, S., Bassi, R., Golan, T., Kramer, D., et al. (2004). Regulation of photosynthetic light harvesting involves intrathylakoid lumen ph sensing by the psbs protein. J. Biol. Chem. 279, 22866-22874. doi: $10.1074 /$ jbc.M402461200

Liguori, N., Campos, S. R. R., Baptista, A. M., and Croce, R. (2019). Molecular anatomy of plant photoprotective switches: The sensitivity of psbs to the environment, residue by residue. J. Phys. Chem. Lett, 10, 1737-1742. doi: $10.1021 /$ acs.jpclett.9b00437

Liu, C., Zhang, Y., Cao, D., He, Y., Kuang, T., and Yang, C. (2008). Structural and functional analysis of the antiparallel strands in the lumenal loop of the major light-harvesting chlorophyll $\mathrm{a} / \mathrm{b}$ complex of photosystem ii (lhciib) by site-directed mutagenesis. J. Biol. Chem. 283, 487-495. doi: 10.1074/jbc.M705736200

Liu, Z., Yan, H., Wang, K., Kuang, T., Zhang, J., Gui, L., et al. (2004). Crystal structure of spinach major light-harvesting complex at 2.72 a resolution. Nature 428, 287-292. doi: 10.1038/nature02373

Lukeš, V., Christensson, N., Milota, F., Kauffmann, H. F., and Hauer, J. (2011). Electronic ground state conformers of $\beta$-carotene and their role in ultrafast spectroscopy. Chem. Phys. Lett. 506, 122-127. doi: 10.1016/j.cplett.2011.02.060

Ma, Y.-Z., Holt, N. E., Li, X.-P., Niyogi, K. K., and Fleming, G. R. (2003). Evidence for direct carotenoid involvement in the regulation of photosynthetic light harvesting. Proc. Natl. Acad. Sci. U.S.A. 100, 4377-4382. doi: 10.1073/pnas.0736959100

Madjet, M. E., Abdurahman, A., and Renger, T. (2006). Intermolecular coulomb couplings from ab initio electrostatic potentials: application to optical transitions of strongly coupled pigments in photosynthetic antennae and reaction centers. J. Phys. Chem. B 110, 17268-17281. doi: 10.1021/jp0615398

Malnoë, A., Schultink, A., Shahrasbi, S., Rumeau, D., Havaux, M., and Niyogia, K. K. (2018). The plastid lipocalin lcnp is required for sustained photoprotective energy dissipation in arabidopsis. Plant Cell. 30, 196-208. doi: 10.1105/tpc.17.00536

Malý, P., Gruber, J. M., van Grondelle, R., and Mancal, T. (2016). Single molecule spectroscopy of monomeric LHCII: Experiment and theory. Sci. Rep. 6, 26230. doi: $10.1038 /$ srep 26230

Mascoli, V., Liguori, N., Xu, P., Roy, L. M., van Stokkum, I. H. M., and Croce, R. (2019). Capturing the quenching mechanism of light-harvesting complexes of plants by zooming in on the ensemble. Chem 5, 2900-2912. doi: 10.1016/j.chempr.2019.08.002

Müh, F., Madjet, M. E.-A., and Renger, T. (2010). Structure-based identification of energy sinks in plant light-harvesting complex II. J. Phys. Chem. B 114, 13517-13535. doi: 10.1021/jp106323e

Müller, M. G., Lambrev, P., Reus, M., Wientjes, E., Croce, R., and Holzwarth, A. R. (2010). Singlet energy dissipation in the photosystem II light-harvesting complex does not involve energy transfer to carotenoids. Chem. Phys. Chem. 11, 1289-1296. doi: 10.1002/cphc.200900852

Müller, P., Li, X.-P., and Niyogi, K. K. (2001). Non-photochemical quenching. a response to excess light energy. Plant Physiol. 125, 1558-1566. doi: $10.1104 /$ pp.125.4.1558

Nicol, L., and Croce, R. (2021). The psbs protein and low ph are necessary and sufficient to induce quenching in the light-harvesting complex of plants lhcii. Sci. Rep. 11, 7415. doi: 10.1038/s41598-021-86975-9

Niyogi, K. K. (2000). Safety valves for photosynthesis. Curr. Opin. Plant Biol. 3, 455-460. doi: 10.1016/S1369-5266(00)00113-8

Novoderezhkin, V., Marin, A., and Grondelle, R., v. (2011). Intra- and intermonomeric transfers in the light harvesting LHCII complex: the redfield-förster picture. Phys. Chem. Chem. Phys. 13, 17093-17103. doi: 10.1039/c1cp21079c

Novoderezhkin, V., Palacios, M. A., Amerongen, H. V., and Grondelle, R. V. (2004). Energy-transfer dynamics in the LHCII complex of higher plants: Modified redfield approach. J. Phys. Chem. B 108, 10363-10375. doi: 10.1021/jp0496001

Ostroumov, E. E., Götze, J. P., Reus, M., Lambrev, P. H., and Holzwarth, A. R. (2020). Characterization of fluorescent chlorophyll charge-transfer states as intermediates in the excited state quenching of light-harvesting complex ii. Photosynth. Res. 144, 171-193. doi: 10.1007/s11120-020-00745-8

Pascal, A. A., Liu, Z., Broess, K., van Oort, B., van Amerongen, H., Wang, C., et al. (2005). Molecular basis of photoprotection and control of photosynthetic light-harvesting. Nature 436, 134-137. doi: 10.1038/nature03795

Polívka, T., and Sundström, V. (2004). Ultrafast dynamics of carotenoid excited states-from solution to natural and artificial systems. Chem. Rev. 104, 2021-2072. doi: 10.1021/cr020674n

Polívka, T., Zigmantas, D., Sundström, V., Formaggio, E., Cinque, G., and Bassi, R. (2002). Carotenoid $s_{1}$ state in a recombinant light-harvesting complex of photosystem ii. Bio Chem. 41, 439-450. doi: 10.1021/bi011589x

Powles, S. B. (1984). Photoinhibition of photosynthesis induced by visible light. Ann. Rev. Plant Physiol. 35, 15-44. doi: 10.1146/annurev.pp.35.060184.000311

Renger, T., Trostmann, I., Theiss, C., Madjet, M. E., Richter, M., Paulsen, H., et al. (2007). Refinement of a structural model of a pigment-protein complex by accurate optical line shape theory and experiments. J. Phys. Chem. B 111, 10487-10501. doi: 10.1021/jp0717241

Ruban, A. V. (2016). Nonphotochemical chlorophyll fluorescence quenching: mechanism and effectiveness in protecting plants from photodamage. Plant Physiol. 170, 1903-1916. doi: 10.1104/pp.15.01935

Ruban, A. V., Berera, R., Ilioaia, C., van Stokkum, I. H. M., Kennis, J. T. M., Pascal, A. A., et al. (2007). Identification of a mechanism of photoprotective energy dissipation in higher plants. Nature 450, 575-578. doi: 10.1038/natu re06262

Ruban, A. V., and Horton, P. (1999). The xanthophyll cycle modulates the kinetics of nonphotochemical energy dissipation in isolated light-harvesting complexes, intact chloroplasts, and leaves of spinach. Plant Physiol. 119, 531-542. doi: 10.1104/pp.119.2.531

Ruban, A. V., Johnson, M. P., and Duffy, C. D. P. (2012). The photoprotective molecular switch in the photosystem ii antenna. Biochim. Biophys. Acta 1817, 167-181. doi: 10.1016/j.bbabio.2011.04.007

Ruban, A. V., and Wilson, S. (2020). The mechanism of non-photochemical quenching in plants: localisation and driving forces. Plant Cell Physiol. 62, 1063-1072. doi: 10.1093/pcp/pcaa155

Saccon, F., Durchan, M., Bína, D., Duffy, C. D., Ruban, A. V., and Polívka, T. (2020). A protein environment-modulated energy dissipation channel in lhcii antenna complex. iScience 23, 101430. doi: 10.1016/j.isci.2020.101430

Sato, R., Ohta, H., and Masuda, S. (2014). Prediction of respective contribution of linear electron flow and pgr5-dependent cyclic electron flow to nonphotochemical quenching induction. Plant Physiol. Bio Chem. 81, 190-196. doi: 10.1016/j.plaphy.2014.03.017

Son, M., Pinnola, A., Bassi, R., and Schlau-Cohen, G. S. (2019). The electronic structure of lutein 2 is optimized for light harvesting in plants. Chem 5, 575-584. doi: 10.1016/j.chempr.2018.12.016

Son, M., Pinnola, A., Gordon, S. C., Bassi, R., and Schlau-Cohen, G. S. (2020). Observation of dissipative chlorophyll-to-carotenoid energy transfer in lightharvesting complex II in membrane nanodiscs. Nat. Commun. 11, 1295. doi: 10.1038/s41467-020-15074-6

Staleva, H., Komenda, J., Shukla, M. K., Šlouf, V., Kaňa, R., Polívka, T., et al. (2015). Mechanism of photoprotection in the cyanobacterial ancestor of plant antenna proteins. Nat. Chem. Biol. 11, 287-291. doi: 10.1038/nchem bio. 1755

Strand, D. D., and Kramer, D. M. (2014). "Control of non-photochemical exciton quenching by the proton circuit of photosynthesis," in Non-Photochemical Quenching and Energy Dissipation in Plants, Algae and Cyanobacteria, eds. B. Demmig-Adams, G. Garab, W. A., III, and U. Govindjee (Dordrecht: Springer), 387-408. doi: 10.1007/978-94-017-9032-1_18

Tavan, P., and Schulten, K. (1987). Electronic excitations in finite and infinite polyenes. Phys. Rev. B 36, 4337. doi: 10.1103/PhysRevB.36.4337

Walla, P. J., Linden, P. A., Ohta, K., and Fleming, G. R. (2002). Excited-state kinetics of the carotenoid s1 state in lhc ii and two-photon excitation spectra of lutein and $\beta$-carotene in solution: efficient car s1-chl electronic energy transfer via hot s1 states? J. Phys. Chem. A 106, 1909-1916. doi: 10.1021/jp0 $11495 \mathrm{x}$ 
Walla, P. J., Yom, J., Krueger, B., and Fleming, G. (2000). Two photon excitation spectrum of lhcii and fluorescence up-conversion after one- and two-photon excitation of the carotenoids. J. Phys. Chem. B 104, 4799-4806. doi: $10.1021 /$ jp9943023

Walters, R. G., Ruban, A. V., and Horton, P. (1994). Higher plant lightharvesting complexes lhciia and lhciic are bound by dicyclohexylcarbodiimide during inhibition of energy dissipation. Eur. J. Bio Chem. 226, 1063-1069. doi: 10.1111/j.1432-1033.1994.01063.x

Wei, T., Balevicius, V., Polívka, T., Ruban, A. V., and Duffy, C. D. P. (2019). How carotenoid distortions may determine optical properties: lessons from the orange carotenoid protein. Phys. Chem. Chem. Phys. 21, 23187-23197. doi: 10.1039/C9CP03574E

Wei, X., Su, X., Cao, P., Liu, X., Chang, W., Li, M., et al. (2016). Structure of spinach photosystem II-LHCII supercomplex at 3.2 åresolution. Nature 534, 69-74. doi: 10.1038/nature18020

Xu, P., Tian, L., Kloz, M., and Crocea, R. (2015). Molecular insights into zeaxanthin-dependent quenching in higher plants. Sci. Rep. 5, 13679. doi: $10.1038 /$ srep 13679
Conflict of Interest: The authors declare that the research was conducted in the absence of any commercial or financial relationships that could be construed as a potential conflict of interest.

Publisher's Note: All claims expressed in this article are solely those of the authors and do not necessarily represent those of their affiliated organizations, or those of the publisher, the editors and the reviewers. Any product that may be evaluated in this article, or claim that may be made by its manufacturer, is not guaranteed or endorsed by the publisher.

Copyright (c) 2022 Gray, Wei, Polivka, Daskalakis and Duffy. This is an open-access article distributed under the terms of the Creative Commons Attribution License (CC $B Y)$. The use, distribution or reproduction in other forums is permitted, provided the original author(s) and the copyright owner(s) are credited and that the original publication in this journal is cited, in accordance with accepted academic practice. No use, distribution or reproduction is permitted which does not comply with these terms. 